Stochastic Systems In Merging Phase Space 
This page intentionally left blank 


\title{
Stochastic Systems In Merging Phase Space
}

\author{
Vladimir S. Koroliuk \\ Institute of Mathematics, National Academy of Sciences, Ukraine
}

Nikolaos Limnios Applied Mathematics Laboratory, University of Technology of Compiègne, France 


\section{Published by}

World Scientific Publishing Co. Pte. Ltd.

5 Toh Tuck Link, Singapore 596224

USA office: 27 Warren Street, Suite 401-402, Hackensack, NJ 07601

UK office: 57 Shelton Street, Covent Garden, London WC2H 9HE

\section{British Library Cataloguing-in-Publication Data}

A catalogue record for this book is available from the British Library.

\section{STOCHASTIC SYSTEMS IN MERGING PHASE SPACE}

Copyright $\odot 2005$ by World Scientific Publishing Co. Pte. Ltd.

All rights reserved. This book, or parts thereof, may not be reproduced in any form or by any means, electronic or mechanical, including photocopying, recording or any information storage and retrieval system now known or to be invented, without written pernission from the Publisher.

For photocopying of material in this volume, please pay a copying fee through the Copyright Clearance Center, Inc., 222 Rosewood Drive, Danvers, MA 01923, USA. In this case permission to photocopy is not required from the publisher.

ISBN $981-256-591-4$ 


\section{Preface}

"... the theory of systems should be built on the methods of simplification and is, essentially, the science of simplification".

Walter Ashby (1969)

The actual problem of systems theory is the development of mathematically justified methods of simplification of complicated systems whose mathematical analysis is difficult to perform even with help of modern computers. The main difficulties are caused by the complexity of the phase (state) space of the system, which leads to virtually boundedless mathematical models.

A simplified model for a system must satisfy the following conditions: (i) The local characteristics of the simplified model are determined by rather simple functions of the local characteristics of original model. (ii) The global characteristics describing the behavior of the stochastic system can be effectively calculated on large enough time intervals. (iii) The simplified model has an effective mathematical analysis, and the global characteristics of the simplified model are close enough to the corresponding characteristics of the original model for application.

Stochastic systems considered in the present book are evolutionary systems in random medium, that is, dynamical systems whose state space is subject to random variations. From a mathematical point of view, such systems are naturally described by operator-valued stochastic processes on Banach spaces and are nowadays known as Random evolution.

This book gives recent results on stochastic approximation of systems by weak convergence techniques. General and particular schemes of proofs for average, diffusion, diffusion with equilibrium, and Poisson approximations of stochastic systems are presented. The particular systems studied here 
are stochastic additive functionals, dynamical systems, stochastic integral functionals, increment processes, impulsive processes. As application we give the cases of absorption times, stationary phase merging, semi-Markov random walk, Lévy approximation, etc.

The main mathematical object of this book is a family of coupled stochastic processes $\xi^{\varepsilon}(t), x^{\varepsilon}(t), t \geq 0, \varepsilon>0$ (where $\varepsilon$ is the small series parameter) called the switched and switching processes. The switched process $\xi^{\varepsilon}(t), t \geq 0$, describes the system evolution, and, in general, is a stochastic functional of a third process. The switching process $x^{\varepsilon}(t), t \geq 0$, also called the driving or modulation process, is the perturbing process or the random medium, and can represent the environment, the technical structure, or any perturbation factor.

The two modes of switching considered here are Markovian and semiMarkovian. Of course, we could present only the semi-Markov case since the Markov is a special case. But we present both mainly for two reasons: the first is that proofs are simpler for the Markov case, and the second that most of the readers are mainly interested by the Markov case.

The switching processes are considered in phase split and merging scheme. The phase merging scheme is based on the split of the phase space into disjoint classes

$$
E=\cup_{k \in V} E_{k}, \quad E_{k} \cap E_{k^{\prime}}=\varnothing, \quad k \neq k^{\prime}
$$

and further merging these classes $E_{k}, k \in V$, into distinct states $k \in V$. So the merged phase space of the simplified model of system is $\hat{E}=V$ (see Figure 4.1). ${ }^{1}$

The transitions (connections) between the states of the original system $S$ are merged to yield the transitions between merged states of the merged system $\hat{S}$. The analysis of the merged system is thus significantly simplified.

It is important to note that the additional supporting system $S^{0}$ with the same phase space $E$ but without connections between classes of states $E_{k}$ is used.

Split the phase space (0.1) just means introducing a new supporting system consisting of isolated subsystems $S_{k}, k \in V$, defined on classes of states $E_{k}, k \in V$. The merged system $\hat{S}$ is constructed with respect to the ergodic property of the support system $S^{0}$.

It is worth noticing that the initial processes in the series scheme contain no diffusion part. Diffusion processes appear only as limit processes.

\footnotetext{
${ }^{1}$ Figures, theorems, lemmas, etc. are numbered by $x . y$, where $\mathrm{x}$ is the number of chapter, and $\mathrm{y}$ is the number of figure, theorem, etc. into the chapter.
} 
The general scheme of proof of weak convergence for stochastic processes in series scheme is the following.

\section{Limit compensating operator:}

1. Construction of the compensating operator $\mathbb{L}^{\varepsilon}$ of the Markov additive process $\xi^{\varepsilon}(t), t \geq 0$.

2. Asymptotic form of $\mathbb{L}^{\varepsilon}$ acting on some kind of test functions $\varphi^{\varepsilon}$.

3. Singular perturbation problem: $\mathbb{L}^{\varepsilon} \varphi^{\varepsilon}=\Psi+\varepsilon \theta^{\varepsilon}$.

II. Tightness:

1. Compact containment condition

$$
\lim _{M \rightarrow \infty} \sup _{0<\varepsilon \leq \varepsilon_{0}} \mathbb{P}\left(\sup _{0<t \leq T}\left|\xi^{\varepsilon}(t)\right| \geq M\right)=0 .
$$

2. Submartingale condition. The process

$$
\eta^{\varepsilon}(t):=\varphi\left(\xi^{\varepsilon}(t)\right)+C_{\varphi} t,
$$

is a nonnegative submartingale with respect to some filtration.

In the case of semimartingales, used in proofs of Poisson approximation results, we follow the same scheme of weak convergence proof, but for their predictable characteristics presented under integral functional forms.

For Markov switching processes, step II is performed in a simpler and more adequate way by using predictable square characteristics of martingale characterization.

It is worth noticing that most of results presented in this book are new, or generalize results published previously by the authors.

The book is organized as follows.

In Chapter 1, we present shortly the basic families of stochastic processes used in this book. More specifically, we present the Markov, semi-Markov processes, and some of their subfamilies, and semimartingales.

In Chapter 2, we present the notions of switching and switched processes via additive functionals of processes with locally independent increments switched by Markov or semi-Markov processes.

In Chapter 3, we present stochastic systems in the series scheme. More specifically, we present the basic results of average and diffusion approximations; proofs are postponed until the next chapters. We start from the simplest case of integral functionals to end by the more complicated case of general additive functionals. 
In Chapter 4, we present results concerning average and diffusion approximations, as in Chapter 3, but with the addition that the state space of the switching process is asymptotically merged (single and double merged). Ergodic and non-ergodic switching processes are considered.

In the next two chapters we present detailed proofs of the results stated in Chapters 3 and 4.

In Chapter 5, we present the algorithmic part of the proofs, realized by the singular perturbing reducible-invertible operator technique. This part corresponds to the finite-dimensional distribution convergence and is performed as in step I above.

In Chapter 6, we give the last step of the proofs concerning the tightness of the probability distribution of the processes. This is performed as in step II above.

In Chapter 7, we give Poisson approximation results for impulsive processes switched by Markov processes and for stochastic additive functionals switched by semi-Markov processes.

The next two chapters include applications of the theory presented in the previous chapters.

In Chapter 8, we present several applications: absorption time distributions, stationary phase merging, superposition of two independent renewal processes, semi-Markov random walks.

In Chapter 9, we present birth-and-death processes, repairable systems, and Lévy approximation of impulsive systems.

Finally, we give some problems to solve. These problems include results stated without proofs in previous chapters, additional results, and some extensions.

Three appendices are also included. The first gives some definitions about weak convergence of stochastic processes. The second gives some known basic theorems on convergence of semimartingales and composed processes needed in proofs. The third includes some additional results concerning intermediate proofs of theorems. Of course, even if these theorems are included in order that this book becomes as autonomous as possible, we encourage the readers to find the corresponding information directly to bibliography.

This book should serve as a textbook for graduate students, postdoctoral seminars or courses for applied scientists and engineers in stochastic approximation of complex systems: queuing, reliability, risk, finance, 
biology systems, etc.

Acknowledgment. Authors express their gratitude to DFG for a financial support of the project 436 UKR 113/70/2-5 and thank particularly Sergio Albeverio of the Institute of Applied Mathematics at University of Bonn, and Yuri Kondratiev of the BiBos Center of the University of Bielelfeld for their hospitality. They are also grateful to the University of Technology of Compiègne for some support and hospitality. They are also grateful to Vladimir Anisimov and Anatoli Skorokhod for several discussions about these problems.

Last, thanks are due also to Esther Tan Leng-Leng, Desk Editor in World Scientific, for her useful collaboration.

Kiev

Compiègne

July 2005
V.S. Koroliuk

N. Limnios 
This page intentionally left blank 


\section{Contents}

Preface v v

1. Markov and Semi-Markov Processes 1

1.1 Preliminaries . . . . . . . . . . . . . . . 1

1.2 Markov Processes . . . . . . . . . . . . . . . . . 2

1.2.1 Markov Chains . . . . . . . . . . . . 2

1.2.2 Continuous-Time Markov Processes . . . . . . . . 6

1.2.3 Diffusion Processes . . . . . . . . . . . . . . 10

1.2.4 Processes with Independent Increments . . . . . . . 11

1.2.5 Processes with Locally Independent Increments . . . 14

1.2.6 Martingale Characterization of Markov Processes . . 15

1.3 Semi-Markov Processes . . . . . . . . . . . . . . . . 19

1.3.1 Markov Renewal Processes . . . . . . . . . . . . 19

1.3.2 Markov Renewal Equation and Theorem . . . . . . 21

1.3.3 Auxiliary Processes . . . . . . . . . . . . . . . . 23

1.3.4 Compensating Operators . . . . . . . . . . . . 24

1.3.5 Martingale Characterization of Markov Renewal Processes . . . . . . . . . . . 25

1.4 Semimartingales $\ldots \ldots \ldots \ldots \ldots \ldots \ldots \ldots$

1.5 Counting Markov Renewal Processes . . . . . . . . . . 28

1.6 Reducible-Invertible Operators . . . . . . . . . . . 31

2. Stochastic Systems with Switching 35

2.1 Introduction . . . . . . . . . . . . . . 35

2.2 Stochastic Integral Functionals $\ldots \ldots \ldots \ldots . \ldots . \ldots 36$

2.3 Increment Processes . . . . . . . . . . . . . . 40 40 
2.4 Stochastic Evolutionary Systems . . . . . . . . . . 43

2.5 Markov Additive Processes . . . . . . . . . . . . . 46

2.6 Stochastic Additive Functionals . . . . . . . . . . . . 47

2.7 Random Evolutions . . . . . . . . . . . . . 50

2.7.1 Continuous Random Evolutions . . . . . . . . . 50

2.7.2 Jump Random Evolutions . . . . . . . . . . . . 54

2.7.3 Semi-Markov Random Evolutions . . . . . . . . . 56

2.8 Extended Compensating Operators . . . . . . . . . . . 59

2.9 Markov Additive Semimartingales . . . . . . . . . . 61

2.9.1 Impulsive Processes . . . . . . . . . . . . . . . 61

2.9.2 Continuous Predictable Characteristics . . . . . . . 63

3. Stochastic Systems in the Series Scheme 67

3.1 Introduction . . . . . . . . . . . . . 67

3.2 Random Evolutions in the Series Scheme . . . . . . . . 68

3.2.1 Continuous Random Evolutions . . . . . . . . . 68

3.2 .2 Jump Random Evolutions . . . . . . . . . . . . 72

3.3 Average Approximation . . . . . . . . . . . . . . 74

3.3.1 Stochastic Additive Functionals . . . . . . . . 74

3.3.2 Increment Processes . . . . . . . . . . . . . . 79

3.4 Diffusion Approximation . . . . . . . . . . . . . . 81

3.4.1 Stochastic Integral Functionals . . . . . . . . 81

3.4.2 Stochastic Additive Functionals . . . . . . . . . 84

3.4.3 Stochastic Evolutionary Systems . . . . . . . 88

3.4.4 Increment Processes . . . . . . . . . . . . . 89

3.5 Diffusion Approximation with Equilibrium . . . . . . . 90

3.5.1 Locally Independent Increment Processes . . . . . 90

3.5.2 Stochastic Additive Functionals with Equilibrium . . 93

3.5.3 Stochastic Evolutionary Systems with Semi-Markov Switching . . . . . . . . . . 97

4. Stochastic Systems with Split and Merging 103

4.1 Introduction . . . . . . . . . . . . . . . . . . 103

4.2 Phase Merging Scheme . . . . . . . . . . . . . . . 104

4.2.1 Ergodic Merging . . . . . . . . . . . . . . . 104

4.2 .2 Merging with Absorption . . . . . . . . . . 110

4.2.3 Ergodic Double Merging . . . . . . . . . . . . . 112

4.3 Average with Merging . . . . . . . . . . . 116 
4.3.1 Ergodic Average . . . . . . . . . . . . . . 117

4.3 .2 Average with Absorption . . . . . . . . . . . . 119

4.3.3 Ergodic Average with Double Merging . . . . . . 120

4.3.4 Double Average with Absorption . . . . . . . . . 121

4.4 Diffusion Approximation with Split and Merging . . . . . 122

4.4.1 Ergodic Split and Merging . . . . . . . . . . 123

4.4.2 Split and Merging with Absorption . . . . . . . . 126

4.4.3 Ergodic Split and Double Merging . . . . . . . . 128

4.4.4 Double Split and Merging . . . . . . . . . . . . 130

4.4.5 Double Split and Double Merging . . . . . . . . 132

4.5 Integral Functionals in Split Phase Space . . . . . . . . 134

4.5 .1 Ergodic Split . . . . . . . . . . . . . . . . . . . 134

4.5.2 Double Split and Merging . . . . . . . . . . 137

4.5.3 Triple Split and Merging . . . . . . . . . . 138

5. Phase Merging Principles 139

5.1 Introduction . . . . . . . . . . . . . . . . 139

5.2 Perturbation of Reducible-Invertible Operators . . . . . . 140

5.2 .1 Preliminaries . . . . . . . . . . . . . 140

5.2.2 Solution of Singular Perturbation Problems . . . . 141

5.3 Average Merging Principle . . . . . . . . . . . . 150

5.3.1 Stochastic Evolutionary Systems . . . . . . . . 151

5.3.2 Stochastic Additive Functionals . . . . . . . . . . . 152

5.3 .3 Increment Processes . . . . . . . . . . . . . . 154

5.3.4 Continuous Random Evolutions . . . . . . . . 156

5.3.5 Jump Random Evolutions . . . . . . . . . . . 157

5.3.6 Random Evolutions with Markov Switching . . . . 159

5.4 Diffusion Approximation Principle . . . . . . . . . . 160

5.4 .1 Stochastic Integral Functionals . . . . . . . . . 161

5.4 .2 Continuous Random Evolutions . . . . . . . . . . 165

5.4 .3 Jump Random Evolutions . . . . . . . . . . . . . 169

5.4.4 Random Evolutions with Markov Switching . . . . 172

5.5 Diffusion Approximation with Equilibrium . . . . . . . 173

5.5.1 Locally Independent Increment Processes . . . . . 174

5.5.2 Stochastic Additive Functionals . . . . . . . . . 175

5.5.3 Stochastic Evolutionary Systems with

Semi-Markov Switching . . . . . . . . . 176

5.6 Merging and Averaging in Split State Space . . . . . . . 182

5.6 .1 Preliminaries . . . . . . . . . . . . . . . 182 
5.6.2 Semi-Markov Processes in Split State Space . . . . 184

5.6 .3 Average Stochastic Systems . . . . . . . . . . 186

5.7 Diffusion Approximation with Split and Merging . . . . . 188

5.7 .1 Ergodic Split and Merging . . . . . . . . . . . . . . 188

5.7 .2 Split and Double Merging . . . . . . . . . . . 189

5.7.3 Double Split and Merging . . . . . . . . . . . 190

5.7.4 Double Split and Double Merging . . . . . . . . . 191

6. Weak Convergence 193

6.1 Introduction . . . . . . . . . . . . . . . . . . . 193

6.2 Preliminaries . . . . . . . . . . . . . . . . . 193

6.3 Pattern Limit Theorems . . . . . . . . . . . . . . . 196

6.3.1 Stochastic Systems with Markov Switching . . . . . 196

6.3.2 Stochastic Systems with Semi-Markov Switching . . 201

6.3.3 Embedded Markov Renewal Processes . . . . . . . 205

6.4 Relative Compactness . . . . . . . . . . . . . . . 209

6.4.1 Stochastic Systems with Markov Switching . . . . . 209

6.4.2 Stochastic Systems with Semi-Markov Switching . . 212

6.4.3 Compact Containment Condition . . . . . . . . . . 213

6.5 Verification of Convergence . . . . . . . . . 216

7. Poisson Approximation 219

7.1 Introduction . . . . . . . . . . . . . . 219

7.2 Stochastic Systems in Poisson Approximation Scheme . . . 220

7.2.1 Impulsive Processes with Markov Switching . . . . 220

7.2.2 Impulsive Processes in an Asymptotic Split Phase Space . . . . . . . . . . . . 225

7.2.3 Stochastic Additive Functionals with Semi-Markov Switching . . . . . . . . . . . . 228

7.3 Semimartingale Characterization . . . . . . . . . 231

7.3.1 Impulsive Processes as Semimartingales . . . . . . . 232

7.3.2 Stochastic Additive Functionals . . . . . . . . . 237

8. Applications I 243

8.1 Absorption Times . . . . . . . . . . . . . . . 243

8.2 Stationary Phase Merging . . . . . . . . . . . . . . . 249

8.3 Superposition of Two Renewal Processes . . . . . . . . 253

8.4 Semi-Markov Random Walks . . . . . . . . . . . 258 
8.4 .1 Introduction . . . . . . . . . . . . 258

8.4.2 The algorithms of approximation for SMRW . . . . . 259

8.4.3 Compensating Operators . . . . . . . . . . . 262

8.4.4 The singular perturbation problem ........ 265

8.4.5 Stationary Phase Merging Scheme ... . . . . 267

9. Applications II 269

9.1 Birth and Death Processes and Repairable Systems . . . . . 269

9.1.1 Introduction . . . . . . . . . . . . . . . 269

9.1.2 Diffusion Approximation . . . . . . . . . . . 270

9.1.3 Proofs of the Theorems . . . . . . . . . . . . . . 272

9.2 Lévy Approximation of Impulsive Processes . . . . . . . . 276

9.2.1 Introduction . . . . . . . . . . . . . . 276

9.2.2 Lévy Approximation Scheme . . . . . . . . . . . 278

9.2.3 Proof of Theorems . . . . . . . . . . . . . . 282

$\begin{array}{ll}\text { Problems to Solve } & 287\end{array}$

Appendix A Weak Convergence of Probability Measures $\quad 301$

A.1 Weak Convergence . . . . . . . . . . . . . . . 301

A.2 Relative Compactness . . . . . . . . . . . . . . . . 303

Appendix B Some Limit Theorems for Stochastic Processes 305

B.1 Two Limit Theorems for Semimartingales . . . . . . . . 305

B.2 A Limit Theorem for Composed Processes . . . . . . . . . 308

Appendix C Some Auxiliary Results 311

C.1 Backward Recurrence Time Negligibility . . . . . . . . . 311

C.2 Positiveness of Diffusion Coefficients . . . . . . . . 312

$\begin{array}{ll}\text { Bibliography } & 315\end{array}$

$\begin{array}{ll}\text { Notation } & 325\end{array}$

$\begin{array}{ll}\text { Index } & 329\end{array}$ 